\title{
Comparisons of static brain-body allometries across vertebrates must distinguish between indeterminate and determinate growth
}

\author{
Tom Froese ${ }^{10}{ }^{1,2 \star}$ and Rainer Froese $\mathbb{(}^{3}$ \\ ARISING FROM M. Tsuboi et al. Nature Ecology \& Evolution https://doi.org/10.1038/s41559-018-0632-1 (2018).
}

We applaud Tsuboi et al. ${ }^{1}$ for assembling the most extensive brainbody mass dataset to date and making it publicly available. However, care must be taken when comparing static allometry between lineages exhibiting indeterminate versus determinate growth. It makes little sense to compare the continuous brain-body growth trajectories of adult fish, reptiles and amphibians with the brain-body weight variability of adult birds and mammals whose growth has ceased. Instead, we agree with Deacon ${ }^{2}$ that "comparing ontogenetic curves is ultimately the only way to provide some assurance that we are not comparing apples and oranges". We therefore doubt that smaller slopes of static allometry in adult birds and mammals, which reflect weight variability across individuals whose brains have ceased growing, can help to explain their encephalization.

In other words, plotting brain-body weight trajectories of individual birds or mammals across adult lifetime gives a slope close to zero, because brain weight will remain largely unchanged even if the individual gets thinner or fatter. Deriving static brain-body allometry slopes of species with determinate growth by regressing across small and large non-growing adult individuals gives slopes larger than 0 , which do not reflect their within-individual trajectories. In contrast, regressing across brain-body weight of indeterminately growing adult individuals, whose brain weight increases with body weight, will tend to result in comparatively larger slopes more likely to reflect their individual brain-body weight growth trajectories. Similar concerns apply to the final phase of development: birds and mammals have a shorter 'slow-growth phase' with smaller slopes because of growth cessation, whereas in other lineages growth continues into adulthood. Therefore, comparisons of brain-body allometry need to be restricted to earlier periods of ontogeny during which all lineages still exhibit growth.

Focusing on development also makes sense because of reduced selection pressure on traits exhibited after reproduction has commenced. As Coolidge and Wynn ${ }^{3}$ put it in the context of human evolution: "What seems clear is that brain growth cannot have occurred simply through selection for more neurons in an adult individual. The process entailed changes in both brain development and physical development. It was not simple, and it was energetically expensive". We believe that differences in energetic constraints explain the finding of Tsuboi et al. ${ }^{1}$ that brain development in birds and mammals has an extended rapid-growth phase. As Tsuboi et al. ${ }^{4}$ also noted, their larger brains can be built and maintained only by a highly efficient metabolism, which is facilitated by low-cost access to oxygen in air-breathing endotherms, where enzymes always work at optimum temperatures. In comparison, ectothermic amphibians and reptiles have optimum body temperatures for only a few hours per day, and gill-breathing animals such as fish pay a high energetic cost for extracting limited amounts of oxygen from water ${ }^{5}$. Release from these energetic constraints in birds and mammals laid the foundation for their encephalization: only these lineages could afford to evolve an extended rapid-growth phase that allows their optimal brain sizes to be both relatively bigger and attained faster.

Received: 8 November 2018; Accepted: 14 August 2019; Published online: 23 September 2019

\section{References}

1. Tsuboi, M. et al. Breakdown of brain-body allometry and the encephalization of birds and mammals. Nat. Ecol. Evol. https://doi.org/10.1038/s41559-0180632-1 (2018).

2. Deacon, T. W. Problems of ontogeny and phylogeny in brain-size evolution. Int. J. Primatol. 11, 237-282 (1990).

3. Coolidge, F. L. \& Wynn, T. The Rise of Homo sapiens: The Evolution of Modern Thinking 2nd edn (Oxford Univ. Press, 2018).

4. Tsuboi, M. et al. Evolution of brain-body allometry in Lake Tanganyika cichlids. Evolution 70, 1559-1568 (2016).

5. Pauly, D. \& Cheung, W. W. L. Sound physiological knowledge and principles in modeling shrinking of fishes under climate change. Glob. Change Biol. 24, e15-e26 (2018).

\section{Acknowledgements}

T.F. was supported by a UNAM-DGAPA-PAPIIT grant (IA104717).

\section{Author contributions}

R.F. proposed the idea, T.F. wrote the first draft of the manuscript, and both T.F. and R.F. finalized the manuscript together.

\section{Competing interests}

The authors declare no competing interests.

\section{Additional information}

Correspondence and requests for materials should be addressed to T.F.

Reprints and permissions information is available at www.nature.com/reprints.

Publisher's note Springer Nature remains neutral with regard to jurisdictional claims in published maps and institutional affiliations.

(c) The Author(s), under exclusive licence to Springer Nature Limited 2019

IInstitute for Applied Mathematics and Systems Research, National Autonomous University of Mexico, Mexico City, Mexico. ${ }^{2}$ Center for the Sciences of Complexity, Universidad Nacional Autónoma de México, Mexico City, Mexico. ${ }^{3}$ GEOMAR Helmholtz-Centre for Ocean Research, Kiel, Germany.

*e-mail: t.froese@gmail.com 